\title{
The optimal diagnostic strategies for patient with coronary artery diseases and stable chest pain syndrome: a cost- effectiveness analysis
}

\author{
Parvin Jafari ${ }^{1}$, Reza Goudarzi ${ }^{2^{*}}$, Mohammadreza Amiresmaeili ${ }^{3}$ and Hamidreza Rashidinejad ${ }^{4}$
}

\begin{abstract}
Background: Numerous invasive and noninvasive diagnostic tests with different cost and effectiveness exist for detection of coronary artery disease. This diversity leads to unnecessary utilization of health services. For this reason, this study focused on the cost-effectiveness analysis of diagnostic strategies for coronary artery disease from the perspective of the health care system with 1-year time horizon.

Results: Incremental cost effectiveness ratios of all strategies were less than the threshold except for the electrocardiography-computed tomography angiography-coronary angiography strategy, and cost of the cardiac magnetic resonance imaging-based strategy was higher than the cost of other strategies. Also, the number of correct diagnosis in the electrocardiography-coronary angiography strategy was higher than the other strategies, and its ICER was 15.197 dollars per additional correct diagnosis. Moreover, the sensitivity analysis found that the probability of doing MRI and sensitivity of the exercise electrocardiography had impact on the results.

Conclusion: The most cost-effective strategy for acute patient is ECG-CA strategy, and for chronic patient, the most cost-effective strategies are electrocardiography-single photon emission computed tomography-coronary angiography and electrocardiography-exercise electrocardiography-coronary angiography. Applying these strategies in the same clinical settings may lead to a better utilization of resources.
\end{abstract}

Keywords: Coronary artery disease, Diagnostic tests, Diagnostic strategies, Cost-effectiveness analysis, Sensitivity analysis

\section{Background}

In the last decade, cardiovascular diseases have become one of the factors which threaten human health [1].One of the most common cardiovascular diseases is coronary artery disease which occurs due to the accumulation of masses of lipids such as cholesterol and fibrous tissue in the form of plaque on the artery walls and which provides problem for the blood flow in the vessels [2]. Economic burden of coronary artery disease on health care

\footnotetext{
* Correspondence: rgoudarzi@yahoo.com

${ }^{2}$ Health Services Management Research Center, Institute for Futures Studies in Health, Kerman University of Medical Sciences, Kerman, Iran Full list of author information is available at the end of the article
}

system is significant. For example, in Australia in 2014, coronary artery disease was responsible for $27 \%$ of the health care spending [3]. Also, in 2016, a UK study reported $£ 62,210$ and $£ 35,549$ cost attributable to coronary artery heart diseases for low- and high-risk patients respectively [4]. An Iranian study in 2017 indicated that cost of this disease was approximately between 4715 and 4908 billion dollars [5].

Every day, a large number of people with chest pain refer to heart centers with near half of them without a real cardiac problem. Hence, correct diagnosis and appropriate treatment of these patients make challenges not only for physicians and hospitals but also for 
governments, health-insurance companies, and health maintenance organizations [6].

According to available guidelines, diagnostic tests for coronary artery disease include the following: electrocardiography (ECG), echocardiography (ECHO), exercise electrocardiography (Ex-ECG), computed tomography coronary angiography (CTA), coronary angiography (CA), cardiac single-photon emission computed tomography (SPECT), stress cardiac magnetic resonance imaging (C-MRI), exercise echocardiography (EX-ECHO), and stress echocardiography (stress ECHO) [7-9]. These tests with having different cost and effectiveness might lead to unnecessary utilization of health services and impose an enormous economic burden on families, health care systems, and government. For this, optimal allocation of health resources has become important issues for the health care system [10]. Economic evaluation is one of the explicit methods for resource allocation. Economic evaluations are widely employed in health policies, including the evaluation of preventive and diagnostic programs, intervention, treatment, and decision-making. The most commonly used form of economic evaluation is the cost-effectiveness analysis [11]. Because there is no information about cost-effectiveness of the diagnostic tests in Iran, the aim of this study was to evaluate the cost effectiveness of seven diagnostic tests including: ECG, ECHO, Ex-ECG, CTA, CA, SPEC $\mathrm{T}$, and $\mathrm{C}$-MRI that are the most common in Iran.

\section{Methods}

This study is a cost-effectiveness analysis from the viewpoint of health care system over a 1-year time horizon.

\section{Strategies}

For the purpose of the present study, nine diagnostic strategies were selected. Relevant data were derived from the medical records (2017-2018) of two Iranian hospitals in 2019. Each of the strategies comprised of two to four diagnostic tests out of seven available tests (ECG, ECHO, EX-ECG, CTA, C-MRI, CA, and SPECT) (see Table 1). All of the strategies started with electrocardiography; however, next steps of the strategy depended on the result of its precedent, i.e., if positive or uncertain result is achieved, strategy will continue. For example, for the patient with chest pain, ECG test is done. If the initial test is positive or uncertain, then $\mathrm{ECHO}$ is performed. If ECHO test is also positive, the patient would be subjected to $\mathrm{CA}$.

\section{Modeling}

Decision tree was used for modeling, which consisted of nine branches, each one representing a unique strategy. All strategies consisted of several sub-branches, and for each of them, costs, effectiveness, and probabilities were
Table 1 Diagnostic test in each strategy

\begin{tabular}{lllll}
\hline Strategy & First test & Second test & Third test & Fourth test \\
\hline 1 & ECG $^{\text {c }}$ & ECho $^{\text {b }}$ & CA $^{\text {a }}$ & \\
2 & ECG & CA & & \\
3 & ECG & SPECT & CA & \\
4 & ECG & ECho & SPECT & CA \\
5 & ECG & Echo & EX-ECG & CA \\
6 & ECG & EX-ECG & CA & \\
7 & ECG & EX-ECG & SPECT & CA \\
8 & ECG & CTA & CA & \\
9 & ECG & $\left.C M R\right|^{9}$ & CA & \\
\hline
\end{tabular}

${ }^{a}$ Invasive coronary angiography

${ }^{\mathrm{b}}$ Echocardiography

'Electrocardiography

${ }^{\mathrm{d}}$ Single-photon emission computed tomography

exercise electrocardiography

${ }^{\text {f}}$ Computed tomography coronary Angiography

${ }^{9}$ Cardiac magnetic resonance imaging

entered into the model. Since all patients underwent electrocardiographic test on arrival at the hospital, it was not included in the modeling, but its cost was calculated for all of the strategies (see Fig. 1).

\section{Parameters}

Model parameters included tests sensitivity, real or false positive probability, cost, and effectiveness. Values of probabilities, costs, and effectiveness were calculated based on available data whereas sensitivities were extracted from previous studies (see Table 2).

\section{Costs}

All costs associated with inpatient and outpatient services were considered from the perspective of the health care system. These included direct medical and nonmedical costs.

Direct medical costs encompassed cost of labor, laboratory, pathology, pharmaceuticals, medical goods and equipment, hospitalization, and diagnostic imaging. Direct non-medical costs included cost of capital depreciation, energy consumption, and administrative affairs.

Direct medical costs were collected by referring to the medical records department using the patient records, and direct non-medical costs were collected from the hospital accounting department. Finally, these costs were calculated for each method and strategy separately.

\section{Effectiveness}

Effectiveness was measured by the number of cases who were correctly diagnosed because angiography is considered a gold standard with $100 \%$ sensitivity [14], and given that all strategies ultimately end to angiography, if the angiographic result was positive, it showed that the person had been correctly diagnosed, and if it was 


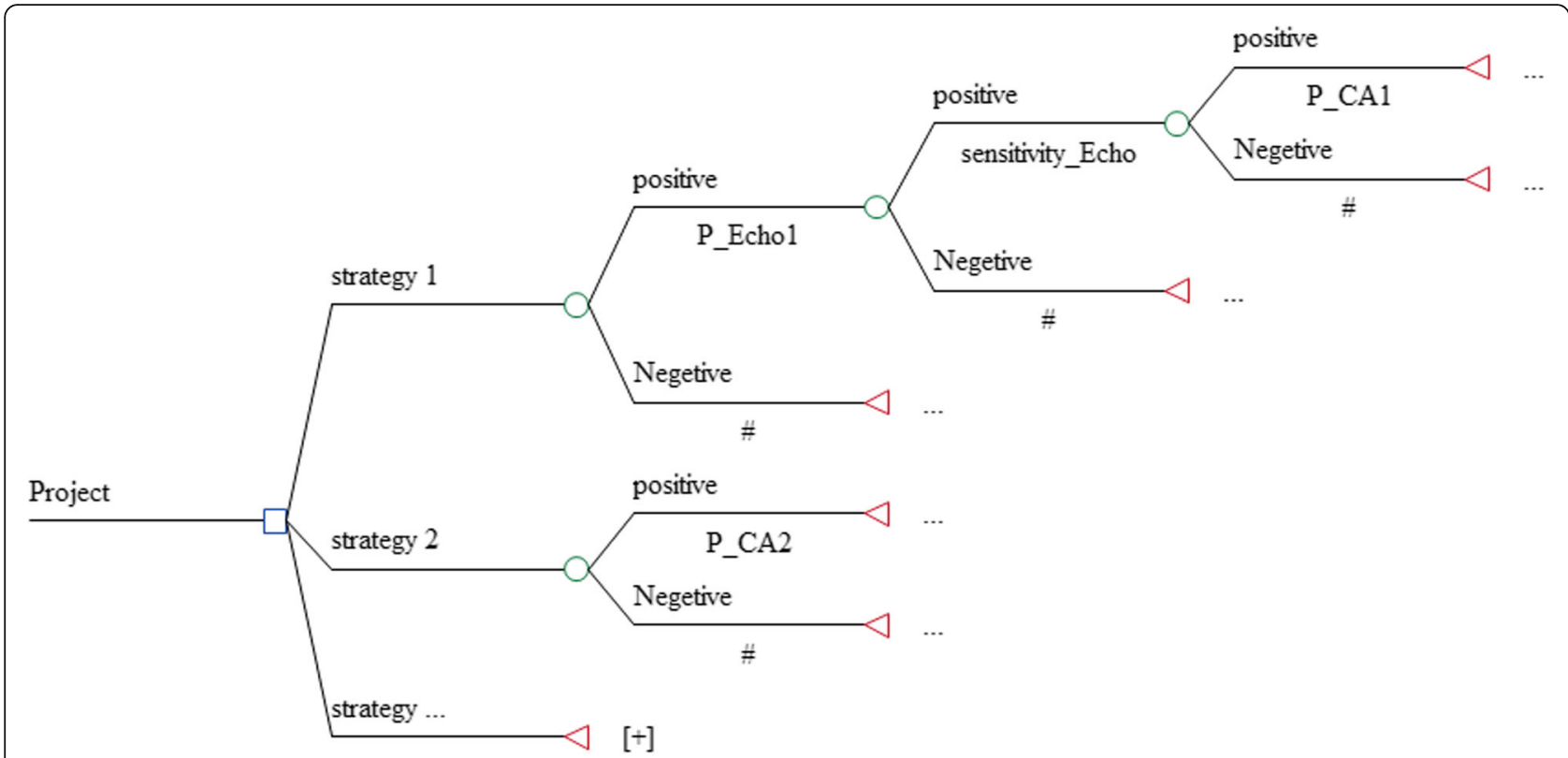

Fig. 1 Decision analytic tree for diagnostic strategies: CA (invasive coronary angiography), C-MRI (cardiac magnetic resonance imaging), CTA (computed tomography coronary angiography), ECHO (echocardiography), Ex-ECG (exercise electrocardiography), SPECT (single-photon emission computed tomography), ECG (electrocardiography)

negative, indicated that the patient had not been correctly diagnosed.

\section{Cost-effectiveness analysis}

Incremental cost effectiveness ratio (ICER) was calculated with the following formula [15]:

$\mathrm{ICER}=\frac{C n-C c}{E n-E c}$ in which:

$\mathrm{Cn}=$ cost of new intervention

En $=$ effect of new intervention

$\mathrm{Cc}=$ cost of current

Ec $=$ effect of current

According to previous studies [12, 14, 16], clinical guidelines, and expert opinions, we found out that strategies 1 and 2 can be used to diagnose cases with acute coronary syndrome and for diagnosing case with chronic coronary syndrome; strategies 3-9 are helpful; hence, diagnostic strategies were analyzed in three categories of total, acute, and chronic.

An expert panel made up of cardiologists, economists, and policymakers suggested the cost of sixth strategy as the baseline with its maximum cost to be regarded as threshold. Therefore, the threshold of the study was set at 2600 \$ per correct diagnosis. All data analysis was performed using decision tree model through TreeAge pro 2011.

\section{Sensitivity analysis}

To test the robustness of the model, the impact of uncertain parameters such as cost, effectiveness, sensitivity, and probabilities on results were assessed. The parameters were analyzed by tornado diagram (Fig. 2); finally, considering the output of the tornado diagram, parameters that had the most effect on the model were analyzed by oneway and two-way sensitivity analysis. Also, probability sensitivity analysis with Monte Carlo simulation was performed for cost parameters using gamma distribution and effectiveness parameters using beta distribution.

\section{Results}

Base case results

Analysis indicated that ECG-Echo-EXECG-CA strategy with $\$ 48.183$ cost and $0.003 \%$ correct diagnosis had the minimum cost and effectiveness, so it was chosen as the current strategy.

ECG-CA strategy with $93.899 \%$ correct diagnoses is the most effective strategy. ECG-CMRI-CA strategy had the highest cost. ICER of ECG-CTA-CA strategy vs strategy 5 is 94.450 dollars per additional case that is above the threshold and is not acceptable. ICER of ECGCA strategy is 15.197 dollars per case, and it is the most cost-effective strategy.

The other information is available in Table 3.

All strategies were located at the northeast of cost effectiveness acceptability plane. The eighth (ECG-TA-CA) strategy has higher cost, and it is placed above of threshold line and therefore is dominated.

\section{Sensitivity results}

All model parameters were considered in Tornado analysis, but the diagram covered only variables which 
Table 2 Input data for decision tree model

\begin{tabular}{|c|c|c|c|c|c|c|c|}
\hline Parameters & Mean & Min & Max & Distribution & $a$ & $\beta$ & Source \\
\hline \multicolumn{8}{|l|}{ Probabilities } \\
\hline $\mathrm{ECHO}^{\mathrm{b}}$ & 0.2 & 0.198 & 0.199 & & & & a \\
\hline SPECT ${ }^{c}$ & 0.291 & 0.166 & 0.418 & & & & a \\
\hline EX-ECG ${ }^{d}$ & 0.146 & 0.057 & 0.210 & & & & a \\
\hline$C T A^{e}$ & 0.031 & 0.031 & 0.031 & & & & a \\
\hline $\mathrm{CMRI}^{\mathrm{f}}$ & 0.636 & 0.636 & 0.636 & & & & a \\
\hline$C A^{g}$ & 0.98 & 0.96 & 1.000 & & & & a \\
\hline \multicolumn{8}{|l|}{ Sensitivity, \% } \\
\hline EX-ECG & 65 & 42 & 92 & & & & [12] \\
\hline $\mathrm{ECHO}$ & 50 & 32 & 68 & & & & [13] \\
\hline CTA & 88 & 83 & 92 & & & & [12] \\
\hline SPECT & 87 & 48 & 88 & & & & [12] \\
\hline CMRI & 89 & 88 & 94 & & & & [12] \\
\hline$C A$ & 100 & 100 & 100 & & & & [12] \\
\hline \multicolumn{8}{|l|}{ Cost (\$) } \\
\hline ECG & 0.793 & 0.026 & 7.401 & Gama & & & \\
\hline EX-ECG & 17.480 & 10.045 & 41.398 & Gama & & & a \\
\hline $\mathrm{ECHO}$ & 33.090 & 8.170 & 220.609 & Gama & & & a \\
\hline CTA & 186.610 & 185.203 & 188.018 & Gama & & & a \\
\hline SPECT & 217.845 & 20.045 & 282.492 & Gama & & & a \\
\hline C-MRI & 54.179 & 53.572 & 55.780 & Gama & & & a \\
\hline CA & 187.686 & 148.034 & 243.199 & Gama & & & a \\
\hline \multicolumn{8}{|l|}{ Effect, \% } \\
\hline Strategy 1 & 19.51 & & & Beta & 0.195 & 0.496 & a \\
\hline Strategy 2 & 96.903 & & & Beta & 0.195 & 0.496 & a \\
\hline Strategy 3 & 40.862 & & & Beta & 0.195 & 0.496 & a \\
\hline Strategy 4 & 3.187 & & & Beta & 0.195 & 0.496 & a \\
\hline Strategy 5 & 1.065 & & & Beta & 0.195 & 0.496 & a \\
\hline Strategy 6 & 20.388 & & & Beta & 0.195 & 0.496 & a \\
\hline Strategy 7 & 5.072 & & & Beta & 0.195 & 0.496 & a \\
\hline Strategy 8 & 2.994 & & & Beta & 0.195 & 0.496 & a \\
\hline Strategy 9 & 63.636 & & & Beta & 0.195 & 0.496 & a \\
\hline
\end{tabular}

${ }^{a}$ Calculated in this study (study's data)

${ }^{\mathrm{b}}$ Echocardiography

'Single-photon emission computed tomography

${ }^{\mathrm{d}}$ Exercise electrocardiography

${ }^{e}$ Computed tomography coronary Angiography

fCardiac magnetic resonance imaging

Invasive coronary angiography

affected results. According to the diagram, probability of C-MRI, CA, and EX-ECG, and sensitivity of CMRI and EX-ECG have the most impact on the results of model. One-way sensitivity analysis indicated that probability of MRI and sensitivity of EXECG influenced the ICERs; however, two-way sensitivity analysis showed that by increasing the probability of MRI, the ninth strategy would become more cost effective, and by increasing the sensitivity of EXECG, the sixth strategy would become more cost effective (Fig. 3).

The cost-effectiveness acceptability curve (Fig. 4) with 1000 iterations showed that the cost-effectiveness probability of strategy 5 (ECG-ECHO-EXECG-CA) under $500 \$$ threshold is $100 \%$. Whereas for the willingness-topay higher than the threshold, the cost-effectiveness 


\section{Tornado Analysis (Net Benefits)}

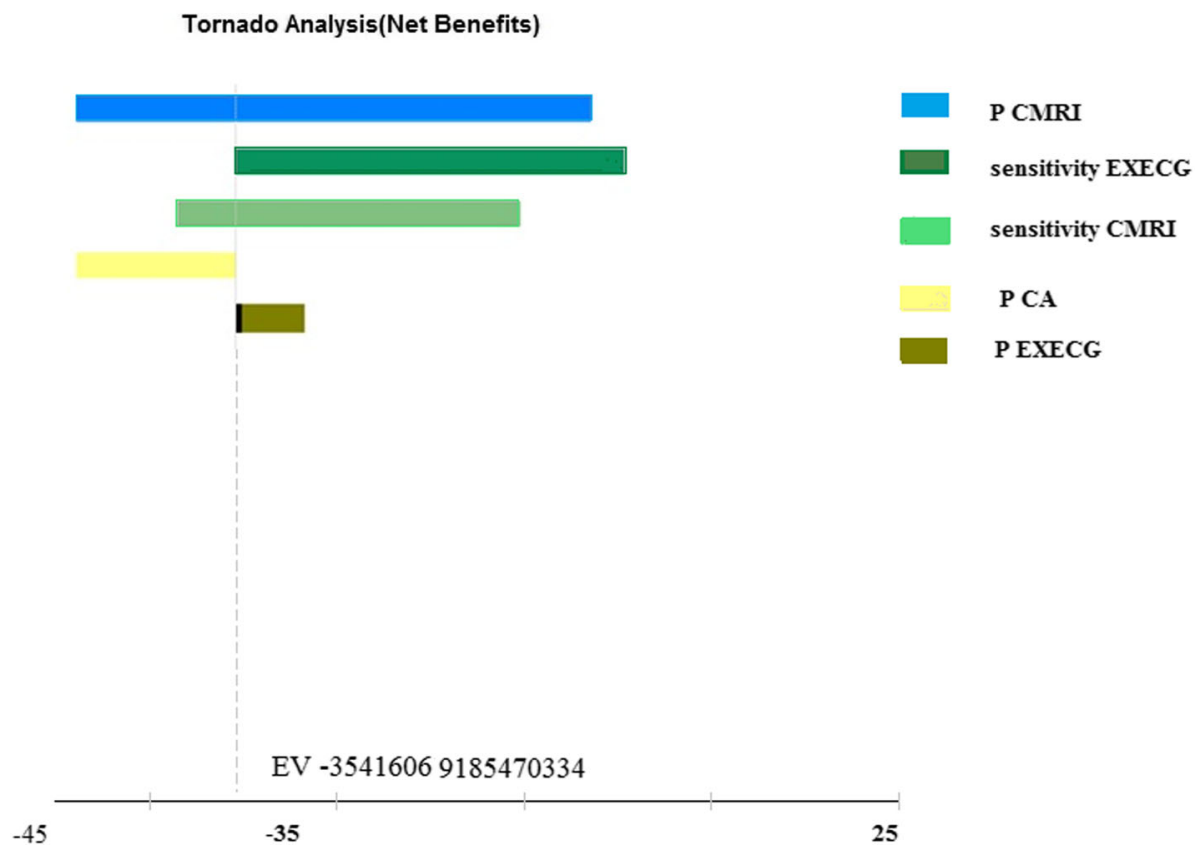

Fig. 2 Tornado diagram: P (probability), CA (invasive coronary angiography), C-MRI (cardiac magnetic resonance imaging), CTA (computed tomography coronary angiography), ECHO (echocardiography), Ex-ECG (exercise electrocardiography), SPECT (single-photon emission computed tomography), ECG (electrocardiography)

Table 3 Base case cost-effectiveness analysis

\begin{tabular}{|c|c|c|c|c|c|c|}
\hline & Cost (\$) & Incremental cost & Effectiveness & Incremental effectiveness & ICER & ACER \\
\hline \multicolumn{7}{|c|}{ Total strategies } \\
\hline Strategy 5 & 48.183 & - & 0.003 & - & - & $16,061.091$ \\
\hline Strategy 4 & 104.076 & 55.893 & 0.043 & 0.04 & 1397.342 & 2420.394 \\
\hline Strategy 7 & 108.378 & 60.195 & 0.137 & 0.342 & 176.009 & 791.084 \\
\hline Strategy 6 & 166.415 & 118.232 & 2.572 & 2.568 & 46.040 & 64.702 \\
\hline Strategy 8 & 255.088 & 206.905 & 0.078 & 0.074 & 2796.019 & 3270.367 \\
\hline Strategy 1 & 364.571 & 316.388 & 1.896 & 1.893 & 167.135 & 192.284 \\
\hline Strategy 3 & 561.280 & 513.097 & 14.488 & 14.484 & 35.425 & 38.741 \\
\hline Strategy 2 & 1475.128 & 1426.945 & 93.899 & 93.895 & 15.197 & 15.709 \\
\hline Strategy 9 & 3450.017 & 3401.834 & 36.020 & 36.017 & 94.450 & 95.780 \\
\hline \multicolumn{7}{|c|}{ Acute patient strategies } \\
\hline Strategy 2 & 364.571 & - & 93.899 & - & - & 3.882 \\
\hline Strategy 1 & 1475.128 & -1110.557 & 1.896 & -92.002 & 12.071 & 778.021 \\
\hline \multicolumn{7}{|c|}{ Chronic patient strategies } \\
\hline Strategy 5 & 48.18327345 & - & 0.003 & - & - & $16,061.09115$ \\
\hline Strategy 4 & 104.0769569 & 55.89368341 & 0.043 & 0.04 & 1397.342085 & 2420.394346 \\
\hline Strategy 7 & 108.3785836 & 60.19531012 & 0.137 & 0.342 & 176.0096787 & 791.0845516 \\
\hline Strategy 6 & 166.4153344 & 118.232061 & 2.572 & 2.568 & 46.04052219 & 64.70269613 \\
\hline Strategy 3 & 561.2803499 & 513.0970764 & 14.488 & 14.484 & 35.42509503 & 38.74105121 \\
\hline
\end{tabular}


Sensitivity Analysis on P_CMRI9 and sensitivityEXECG (Net Bene fit, WTP= 2600\$)

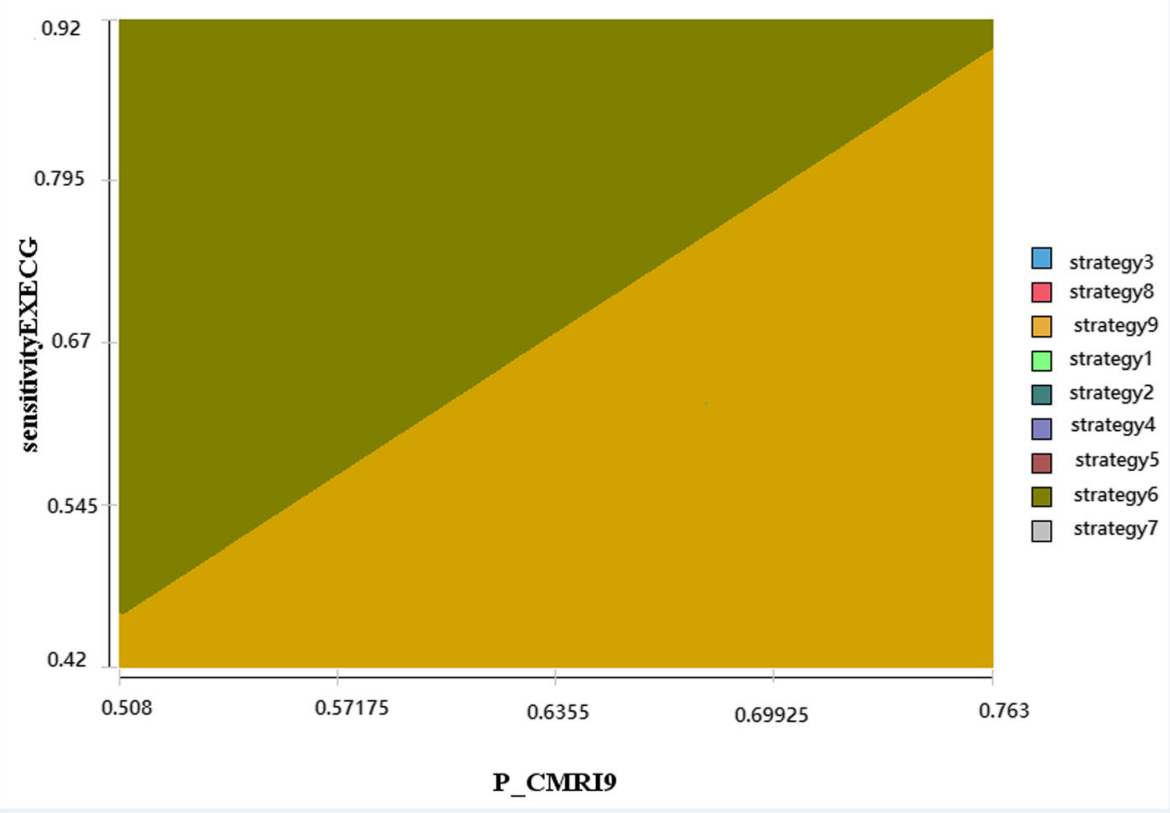

Fig. 3 Two-way sensitivity analysis: two-way sensitivity analysis between sensitivity EX-ECG, P, and CMRI

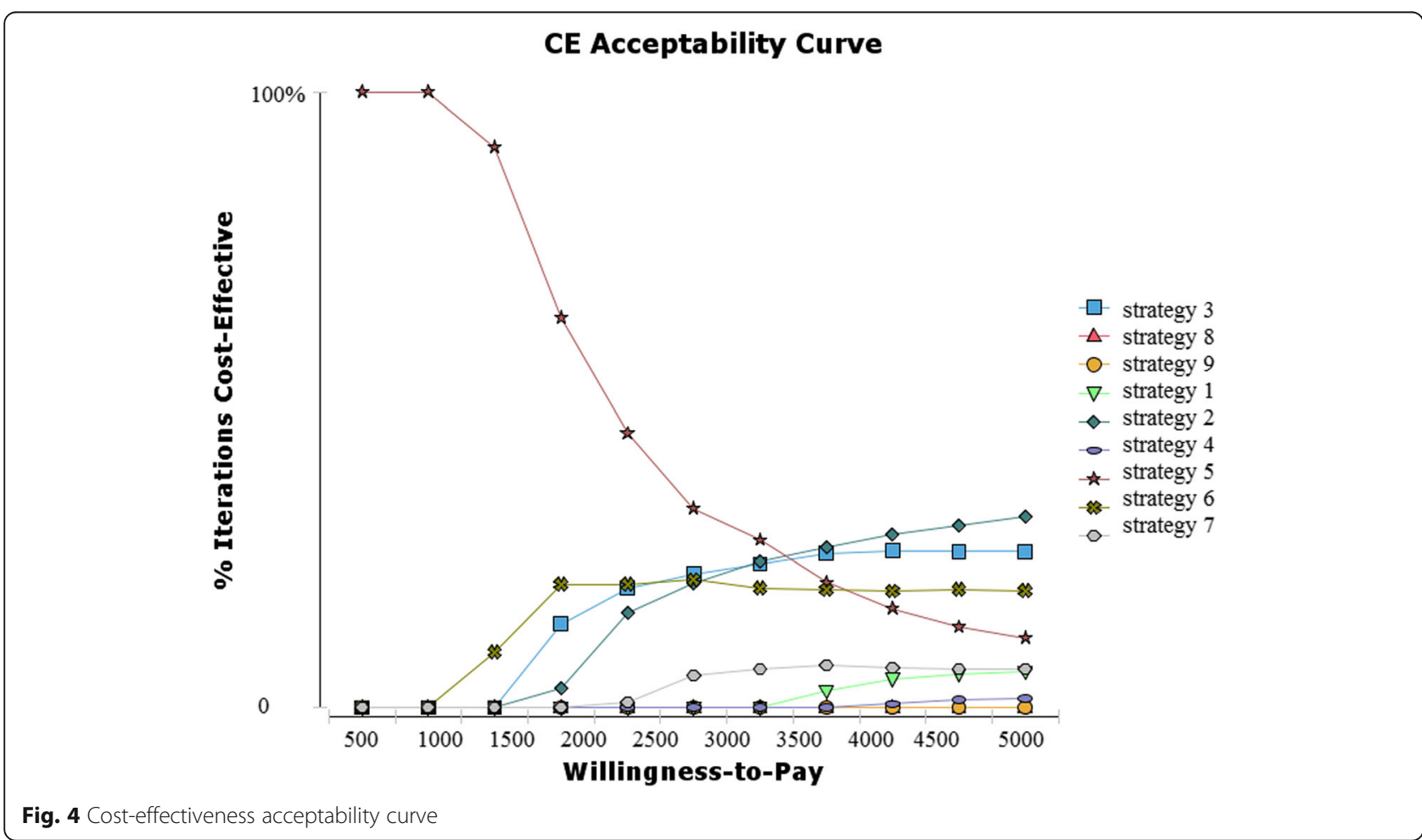


probability of strategy 2 (ECG-CA), strategy 3 (ECGSPECT-CA), and strategy 5 is the highest.

\section{Discussion}

This study was done in two Iranian hospitals during 2017-2018, to evaluate and compare the cost and effectiveness of nine diagnostic strategies for coronary artery disease with chest pain. Based on the results, the SPECT test had the highest cost per case, followed by CTA, MRI, ECHO, EX-ECG, and ECG, respectively. Similarly, Boldt et al. [17] and Min et al. [16] showed that the highest cost belongs to SPECT. Zacharias et al. (2015) and Zacharias et al. (2016) indicated that the cost of ECHO was less than the cost of EXECG which contradicts the present study $[18,19]$.

The MRI-based strategy with the cost of $\$ 3450.017$ was more expensive than the others followed by the SPECT-based strategy and CTA-based strategy respectively. Likewise, Min et al. showed that CTA-based strategy was costlier than EXECG-based strategy. Bertoldi et al.'s study indicated that cardiac MRI-based strategies and SPECT-based strategies had the highest costs and can be used depending on threshold; the study by Walker et al. showed that the cost of the ninth strategy (MRI), which was $\$ 18,284$, is greater than the cost of the SPECT-based strategy [12, 14, 20]. Moschetti et al., Thom et al., and Boldt et al. also depicted that C-MRI is costly but can be used as a good option to diagnose people with a high probability of coronary artery disease and to reduce angiography $[17,21,22]$. In contrast, Min et al. [20] showed that ECG-CA strategy, at $\$ 14,003$, is more expensive than other strategies, and the study by Genders et al. [23] indicated ECG-CTA-CA strategy was less expensive.

According to the results, it is clear that the cost of tests and diagnostic strategies in Iran is lower than the costs in other countries because of the different types of medical insurances that the government provided for people, and the other reason is because of the majority of hospital staffs have low salary.

In this study, the effectiveness of the second strategy (ECG-CA) was 93.899\%, which is higher than the other strategies, followed by MRI-based strategy and SPECTbased strategy. Similarly, Thom et al. showed that rate of correct diagnosis by MRI and ECHO was $80 \%$ and $75 \%$ respectively [22]. Hamilton et al. showed the rate of correct diagnosis by CTA and EXECG were 26\% and 51\% respectively [24]. Similar to the findings of current study, Sharples et al. also indicated that the SPECT-based strategy had $83 \%$ correct diagnosis [25].

To sum up, for acute patient's strategies, the cost and effectiveness of the second strategy (ECG-CA) were significantly higher than ECG-ECH-CA strategy. ICERs indicate that the first strategy generates $\$ 12.071$ more cost per correct diagnosis. Therefore, the second strategy is more cost-effective for patients with acute coronary artery disease.

However, the finding of cost-effectiveness analysis of chronic patients' strategies suggested that the ECGECHO-SPECT-CA and ECG-EX ECG-SPECT-CA strategies were not cost effective and therefore not acceptable because of their higher ICER and ACER. Strategies 3 (ECG-SPECT-CA) and 6 (ECG-EXECG-CA) had an appropriate ICER. Based on the results, it can be concluded that strategies 3 and 6 are more appropriate for people with lower risk and emerged as the most effective strategy.

The American Heart Association's latest guidelines for diagnosis and management of angina made more traditional recommendations for EXECG as the best first line compared to ECHO and SPECT or CTA [12].

In this study, ECG-CTA-CA strategy had high cost. Because in Iran the threshold for a correct diagnosis is low, this strategy is not cost-effective. Although Min et al. study's indicated that the eighth strategy even with ICER \$ 17516 per patient is most cost effective, and also, the studies of Priest et al., Joseph et al., Hamilton et al., and Min et al. showed that the ECG-CTA-CA strategy is the most cost-effective strategy $[16,20,24$, $26,27]$.

A possible limitation of this study was that we did not include all diagnostic strategies and only analyzed strategies which are more common in Iran. Another limitation of the present study is the fact that test sensitivity was extracted from other studies which may be different from the real one. This study was carried out from economic aspect so it might have different results according to the patient's situation. Given that the study did not assess the clinical outcomes, three important concepts are lacking such as the quality-adjusted life years (QALY) assessment, standard gambling (SG), and timetrade off (TTO) concept.

Despite these limitations, to our knowledge, this study is the first study to evaluate the cost-effectiveness of coronary artery disease diagnostic tests in Iran and also this research has analyzed the diagnostic strategies of coronary artery disease in general as well as in two groups of acute and chronic patient strategies.

\section{Conclusion}

This study indicated that all strategies except CTAbased strategy are cost-effective, but the ECG-CA strategy is the most cost-effective strategy for acute patients. For chronic patients, ECG-SPECT-CA and ECG-EX ECG-CA strategies are the best choices. Due to the limited resources in the health care system, applying these strategies to patient in the same clinical setting may lead to a better utilization of resources. Strategy 9 (ECG- 
CMRI-CA) in high threshold may lead to early diagnosis of the disease and thereby saving resource. To summarize, it is recommended to consider economic issues as well a clinical issues for choosing diagnostic strategies, and in the same condition, the cost effectiveness of the strategies should be the basis of choice. Due to the difference in cost effectiveness of diagnostic strategies in Iran compared with other countries, these results should be included in developing local clinical guidelines.

\section{Abbreviations}

ICER: Incremental cost-effectiveness ratio; ACER: Average cost-effectiveness ratio; ECG: Electrocardiography; EX-ECG: Exercise electrocardiography; SPEC $\mathrm{T}$ : Single-photon emission computed tomography; ECHO: Echocardiography; CTA: Computed tomography coronary angiography; CMRI: Cardiac magnetic resonance imaging; CA: Invasive coronary angiography; P: Probability;

QALY: Quality-adjusted life years; SG: Standard gambling; TTO: Time-trade off

\section{Acknowledgements}

Special thanks to all hospitals staffs that providing these data for this study.

\section{Authors' contributions}

PJ: data collection, data analysis, and providing draft version of the paper RG: idea conception, data analysis, and editing draft version of the paper. MA: data analysis and editing draft version of the paper. HR: data analysis and editing draft version of the paper. All authors read and approved the final manuscript.

\section{Funding}

None

\section{Availability of data and materials}

The datasets used and/or analyzed during the current study are available from the corresponding author on reasonable request.

\section{Ethics approval and consent to participate}

Evaluated by: Kerman University of Medical Sciences, approval date: May 25, 2019, and reference number is IR.KMU.REC.1398.117. Consent to participate is not applicable.

\section{Consent for publication}

Not applicable.

\section{Competing interests}

We have no conflict of interest.

\section{Author details}

${ }^{1}$ Social Determinants of Health Research Center, Institute for Futures Studies in Health, Kerman University of Medical Sciences, Kerman, Iran. ${ }^{2}$ Health Services Management Research Center, Institute for Futures Studies in Health, Kerman University of Medical Sciences, Kerman, Iran. ${ }^{3}$ Department of Health Management and Economics, Faculty of Management and Medical Informatics, Kerman University of Medical Sciences, Kerman, Iran.

${ }^{4}$ Cardiovascular Research center,institute of basic and clinical physiology science., Kerman University of Medical Sciences, Kerman, Iran.

Received: 5 July 2020 Accepted: 20 October 2020

Published online: 23 November 2020

\section{References}

1. Ramezani Y, Mobasheri M, Moosavi SG et al (2011) Exposure rate of cardiovascular risk factors among clients of health-care clinics in Kashan Autumn 2010. J Shahrekord Univ Med Sci 13(2):76-82.

2. Salehi N, Nilchi AR (2015) Automatic diagnosis of coronary arteries in heart angiogram images using tree tree tracking of three-dimensional structures scientific forums. Mashhad Ferdowsi University, Mashhad, pp 139-144
3. McCreanor V, Graves N, Barnett A et al (2018) A systematic review and critical analysis of cost-effectiveness studies for coronary artery disease treatment. Research. 7:77

4. Asaria M, Walker S, Palmer S et al (2016) Using electronic health records to predict costs and outcomes in stable coronary artery disease. Heart. https:// doi.org/10.1136/heartjnl-2015-308850

5. Raghfar H, Sargazi N, Mehraban S et al (2018) The economic burden of coronary heart disease in Iran: a bottom-up approach in 2014. J Ardabil Univ Med Sci 18(3):341-356

6. Bassan R (2002) Chest pain units: a modern way of managing patients with chest pain in the emergency department. Arq Bras Cardiol 79:203-209

7. Joseph J, Velasco A, Hage FG (2018) Guidelines in review: comparison of ESC and ACC/AHA guidelines for the diagnosis and management of patients with stable coronary artery disease. J Nucl Cardiol 25(2):509-515

8. Goodacre S, Thokala P, Carroll C et al (2013) Systematic review, metaanalysis and economic modelling of diagnostic strategies for suspected acute coronary syndrome. Health Technol Assess 17(1):v-vi 1-188

9. Montalescot G, Sechtem U, Achenbach S et al (2013) 2013 ESC guidelines on the management of stable coronary artery disease: the Task Force on the management of stable coronary artery disease of the European Society of Cardiology. Eur Heart J 34(38):2949-3003

10. Akbari Sari A, Gholami M (2018) Study of the relationship between health transformation plan and amount of Cathlab room procedures in three hospitals, Tehran, Iran. Res Med 42(3):137-143

11. Husereau D, Drummond M, Petrou S et al (2013) Consolidated health economic evaluation reporting standards (CHEERS) statement. Cost Effect Resour Allocation 11(1):6

12. Bertoldi EG, Stella SF, Rohde LE et al (2016) Long-term cost-effectiveness of diagnostic tests for assessing stable chest pain: modeled analysis of anatomical and functional strategies. Clin Cardiol 39(5):249-256

13. Dresden S, Mitchell P, Rahimi L et al (2014) Right ventricular dilatation on bedside echocardiography performed by emergency physicians aids in the diagnosis of pulmonary embolism. Ann Emerg Med 63(1):16-24

14. Walker S, Girardin F, McKenna C et al (2013) Cost-effectiveness of cardiovascular magnetic resonance in the diagnosis of coronary heart disease: an economic evaluation using data from the CE-MARC study. Heart. https://doi.org/10.1136/heartjnl-2013-303624

15. Fox Rushby J (2005) Economic evaluation. McGraw-Hill Education (UK), England

16. Min JK, Gilmore A, Budoff MJ et al (2010) Cost-effectiveness of coronary CT angiography versus myocardial perfusion SPECT for evaluation of patients with chest pain and no known coronary artery disease. Radiology. 254(3):801-808

17. Boldt J, Leber AW, Bonaventura K et al (2013) Cost-effectiveness of cardiovascular magnetic resonance and single-photon emission computed tomography for diagnosis of coronary artery disease in Germany. J Cardiovasc Magn Reson 15(1):30

18. Zacharias K, Ahmadvazir S, Ahmed A et al (2015) Relative diagnostic prognostic and economic value of stress echocardiography versus exercise electrocardiography as initial investigation for the detection of coronary artery disease in patients with new onset suspected angina. IJC Heart VasC 7:124-130

19. Zacharias K, Ahmed A, Shah BN et al (2016) Relative clinical and economic impact of exercise echocardiography vs. exercise electrocardiography, as first line investigation in patients without known coronary artery disease and new stable angina: a randomized prospective study. Eur Heart J Cardiovasc Imaging 18(2):195-202

20. Min JK, Gilmore A, Jones EC et al (2017) Cost-effectiveness of diagnostic evaluation strategies for individuals with stable chest pain syndrome and suspected coronary artery disease. Clin Imaging 43:97-105

21. Moschetti K, Muzzarelli S, Pinget C et al (2012) Cost evaluation of cardiovascular magnetic resonance versus coronary angiography for the diagnostic work-up of coronary artery disease: application of the European Cardiovascular Magnetic Resonance registry data to the German, United Kingdom, Swiss, and United States health care systems. J Cardiovasc Magn Reson 14(1):35

22. Thom H, West NE, Hughes $V$ et al (2014) Cost-effectiveness of initial stress cardiovascular MR, stress SPECT or stress echocardiography as a gate-keeper test, compared with upfront invasive coronary angiography in the investigation and management of patients with stable chest pain: mid-term outcomes from the CECaT randomised controlled trial. BMJ Open 4(2): e003419 
23. Genders TS, Petersen SE, Pugliese F et al (2015) The optimal imaging strategy for patients with stable chest pain a cost-effectiveness analysis. Ann Intern Med 162(7):474-484

24. Hamilton-Craig C, Fifoot A, Hansen M et al (2014) Diagnostic performance and cost of $\mathrm{CT}$ angiography versus stress ECG - a randomized prospective study of suspected acute coronary syndrome chest pain in the emergency department (CT-COMPARE). Int J Cardiol 177(3):867-873

25. Sharples L, Hughes V, Crean A et al (2007) Cost-effectiveness of functional cardiac testing in the diagnosis and management of coronary artery disease: a randomised controlled trial. The CECaT trial. Health Technol Assess 11(49):1-115.

26. Ladapo JA, Jaffer FA, Hoffmann U et al (2009) Clinical outcomes and costeffectiveness of coronary computed tomography angiography in the evaluation of patients with chest pain. J Am Coll Cardiol 54(25):2409-2422

27. Priest VL, Scuffham PA, Hachamovitch R et al (2011) Cost-effectiveness of coronary computed tomography and cardiac stress imaging in the emergency department: a decision analytic model comparing diagnostic strategies for chest pain in patients at low risk of acute coronary syndromes. JACC Cardiovasc Imaging 4(5):549-556

\section{Publisher's Note}

Springer Nature remains neutral with regard to jurisdictional claims in published maps and institutional affiliations.

\section{Submit your manuscript to a SpringerOpen ${ }^{\circ}$ journal and benefit from:}

- Convenient online submission

- Rigorous peer review

- Open access: articles freely available online

High visibility within the field

- Retaining the copyright to your article

Submit your next manuscript at $\boldsymbol{\nabla}$ springeropen.com 